\title{
EL SISTEMA EDUCATIVO PARAGUAYO ANTE LOS DESAFÍOS SOCIALES DEL SIGLO XXI: UNA EVOLUCIÓN HISTÓRICA HACIA EL PROGRAMA METAS EDUCATIVAS 2021
}

\section{THE PARAGUAYAN EDUCATIONAL SYSTEM FACING THE SOCIAL CHALLENGES OF THE $21^{\text {st }}$ CENTURY: A HISTORICAL EVOLUTION TOWARDS THE EDUCATIONAL GOALS PROGRAM 2021}

\author{
Carlos Novella García
}

\begin{abstract}
In 2011, the Organization of Ibero-American States for Education and Culture assumed the responsibility of coordinating actions and monitoring a very ambitious educational program called "Educational Goals 2021: the education we want for the bicentennial generation". This article pretends to know the historical evolution of the Paraguayan educational system towards this program and analyze the progress and achievements of Paraguay, one of the signatory countries of this project. For this analysis we use national and international reports that allow us to know in detail and reliability the results of the application and development of the eleven goals of the educational program in the Paraguayan educational context. However, by extension of this article we focus on three of these eleven goals achieving very relevant results regarding the achievements and absences of the objectives of the goals analyzed. The data reflect a considerable advance in educational policies from 2011 to the present, although there are still notable shortcomings in aspects related, mainly, to the training, selection and strengthening of the teaching profession. It is necessary to point out that the educational statistical data to which we have had access was very limited since the last accessible MEC report from Paraguay is from 2011, so we had to resort to external reports.
\end{abstract}

Key words: educational goals 2021, history, education system, teaching, evaluation.

\section{RESUMEN}

En el año 2011 la Organización de Estados Iberoamericanos para la Educación y la Cultura asumió la responsabilidad de coordinar las acciones y realizar el seguimiento de un programa educativo muy ambicioso llamado "Metas Educativas 2021: la educación que queremos para la generación de los bicentenarios". Este artículo pretende conocer la evolución histórica del sistema educativo paraguayo hacia este programa y analizar los avances y logros alcanzados por Paraguay, uno de los países firmantes de este proyecto. Para dicho análisis nos servimos de informes nacionales e internacionales que nos permitieran conocer con detalle y fiabilidad los resultados de la aplicación y desarrollo de las once metas del programa en materia educativa en el contexto educativo paraguayo. No obstante, por extensión de este artículo nos centramos en tres de estas once metas consiguiendo resultados muy relevantes respecto a los logros y a las ausencias de los objetivos de las metas analizadas. Los datos reflejan un avance considerable de las políticas educativas desde el 2011 hasta la actualidad aunque siguen existiendo carencias notables en aspectos relacionados, principalmente, con la formación, la selección y el fortalecimiento de la profesión docente. Es necesario apuntar que los datos estadísticos educativos a los que hemos tenido acceso fueron muy 
limitados ya que el último informe del MEC de Paraguay accesible es del 2011 por lo que tuvimos que recurrir a informes externos.

Palabras clave: metas educativas 2021, historia, sistema educativo, docencia, evaluación.

Fecha de recepción: 28 de agosto de 2018

Fecha de aceptación: 29 de enero de 2019 


\section{INTRODUCCIÓN}

En este artículo se pretende mostrar el grado de alcance de los objetivos marcados en el acuerdo establecido en 2010 entre los países asistentes a la XX Conferencia Iberoamericana de Ministros de Educación y a la XX Cumbre Iberoamericana de Jefas y Jefes de Estado y de Gobierno y firmantes del Programa "Metas Educativas 2021: la educación que queremos para la generación de los Bicentenarios", celebrada en Mar de Plata (Argentina) los días 3 y 4 de diciembre de 2010. Como afirma Marchesi (2009) recoge un compromiso común ya de la XVIII Cumbre Iberoamericana de Educación celebrada en El Salvador en 2008 con

La voluntad de acordar entre todos los países iberoamericanos unas metas educativas comunes con el fin de situar sus sistemas educativos entre los más avanzados del mundo y lograr que la generación de los bicentenarios sea la mejor formada de la historia. (p. 88).

La Cumbre del 2008 incorporó en su Declaración de El Salvador el acuerdo de

Fortalecer las políticas educativas y culturales, tendientes a asegurar el derecho a la educación de calidad desde la primera infancia, la cobertura universal y gratuita de la primaria y secundaria y a mejorar las condiciones de vida y las oportunidades reales de las y los jóvenes, que permitan su crecimiento integral para lograr mayores niveles de inclusión y desarrollo social en nuestros países y avanzar en la consolidación del Espacio Iberoamericano del Conocimiento, en el marco de las Metas Educativas 2021. (Marchesi, 2009, p. 88)

Es un buen momento, por tanto, para reflexionar sobre estos avances en uno de los países más prometedores y emergentes de la zona en los últimos años como es Paraguay, uno de los países firmantes de dicho acuerdo. No solamente se trata de comprobar y contrastar lo acordado con lo conseguido sino también establecer posibles nuevas metas que pueden plantearse para completar lo ya logrado. Este programa incorporaba 11 metas educativas a desarrollar en función de cada país estableciéndose como momentos concretos de evaluación de los niveles de logro el 2015 y el 2021. En este mismo acuerdo se designó a la Organización de Estados Iberoamericanos (OEI) como la responsable de coordinar y evaluar el proceso de seguimiento y el del logro de las metas en los países firmantes. En este sentido esta organización elaboró una primera evaluación en 2015 donde aparecen las metas educativas generales y específicas junto a sus indicadores y sus niveles de logro. En síntesis podríamos destacar atendiendo a su informe "Miradas sobre la Educación en Iberoamérica" de 2016 que en los países firmantes ha aumentado la oferta de plazas de educación infantil para niños entre los 0 y los 6 años, la mejora de la calidad formativa y la cualificación profesional de los educadores infantiles pero con la salvedad de que estos avances no se han producido de forma equitativa en la totalidad de los países. Por lo que respecta a la cobertura de la educación primaria, es destacable que los dos tercios de los países iberoamericanos tienen tasas superiores al 90\% llegando a ser el 95\% de nivel de logro esperado para 2015 en siete de ellos. En relación a la calidad educativa y el uso de las TIC como herramienta pedagógica, el informe indica que la evolución es positiva comparando el 2015 con los años anteriores en la ratio alumnoordenador. (OEI, 2016) Aunque comprobamos que son datos esperanzadores el ritmo de avance en los países miembro hacia las metas pactadas está siendo muy desigual. Ante esta situación en este mismo informe se proponen una serie de medidas de mejora necesarias de cara al 2021 según la OEI (2016):

- Ampliar la oferta de educación técnico profesional para aquellos alumnos que terminan la educación secundaria. 


\begin{abstract}
- Mantener el esfuerzo que se viene realizando en educación secundaria baja para alcanzar tasas de matriculación que den cobertura a la práctica totalidad de los alumnos.

- Avanzar en un mejor conocimiento de la situación educativa de los colectivos más vulnerables (minorías étnicas, poblaciones originarias y afrodescendientes) mejorando las estadísticas relativas a estos indicadores, que proporcionan información sobre uno de los aspectos esenciales de la calidad que es lograr la igualdad educativa y superar toda formación de discriminación.

- Ampliar la mirada más allá de los plazos originalmente previstos e iniciar una reflexión sobre las prioridades y estrategias para la concreción de las Metas en el periodo 2021-2030 (...) planteamiento coincidente con el adoptado por la UNESCO en la Agenda de la Educación 2013. (pp. 8-9)
\end{abstract}

Paraguay es un país con cerca de 7 millones de habitantes y está situado en la zona central de América del Sur siendo su capital Asunción. Su distribución territorial se divide en 17 departamentos y un distrito capital. Al sureste, sur y suroeste limita con Argentina, al este con Brasil y con Bolivia por el norte. Es uno de los países más pequeños y poblados de América del Sur. La Constitución de la República del Paraguay de 20 de junio de 1992, que dio fin a la dictadura del General Alfredo Stroessner del 1954 al 1989, declara que es un país bilingüe siendo los idiomas oficiales el español y el guaraní. Fue miembro fundador del Mercosur y se define como Estado soberano, democrático y aconfesional.

Según informe elaborado por el Banco Interamericano para el Desarrollo y publicado en 2018 con el título "Paraguay: Rutas para el desarrollo", es una de las economías de la región que más ha crecido en la última década. En concreto el informe especifica que entre 2004 y el 2016 su PIB creció en promedio un $4,7 \%$ por año, situándose por encima de la media del resto de América Latina y el Caribe. Mientras otros países durante el 2015 y 2016 desaceleraban su crecimiento, la economía de Paraguay creció alrededor de tres puntos por encima cada uno de esos años. La agricultura, la ganadería y la electricidad han sido las fuentes de crecimiento impulsoras de la economía paraguaya. Este crecimiento económico ha logrado reducir la pobreza en el país aunque los datos siguen siendo altos y preocupantes. La escolarización ha logrado alcanzar unos niveles prometedores (lo analizaremos más detalladamente en unos de los apartados de este artículo) como también la esperanza de vida produciéndose los mayores incrementos en las zonas rurales y entre las mujeres. Aunque la calidad de vida ha mejorado las desigualdades entre las zonas rurales y las urbanas es considerable y con mayor porcentaje a las poblaciones indígenas. (Almeida, Bastos, Quijada y Acevedo, 2018).

\title{
1. LA EVOLUCIÓN HISTÓRICA DEL SISTEMA EDUCATIVO DE PARAGUAY DESDE 1811 HASTA LA ACTUALIDAD
}

Como hemos visto en el anterior apartado, Paraguay, disfruta de una joven democracia que sin duda ha afectado en su evolución histórica educativa. Es importante, antes de analizar y contrastar lo logrado de las metas educativas, situar esos avances en los precedentes históricos educativos desde la independencia de España en 1811 con el fin de comprender mejor los niveles desde los que partía hasta lo que ha conseguido en la actualidad.

El sistema educativo paraguayo actual puede considerarse relativamente joven aunque podemos avanzar que la última ley educativa data de 1998 (ley General de Educación no 1264) y no ha tenido una profunda renovación en su articulado desde ese mismo año. Según informe de OEI (1994)

La primera forma de gobierno adoptada en educación fue de carácter colegiado ya que la Junta Superior Gubernativa, que en un documento memorable dictado el 15 de febrero de 1812 la 
"Instrucción para el maestro de primeras letras", y en el Oficio dirigido al Cabildo de Asunción el 10 de marzo, para la difusión general de aquella. (p. 3).

\subsection{LA EDUCACIÓN PARAGUAYA ENTRE 1814 A 1954}

Durante el periodo de 1814 al 1840 bajo el gobierno del dictador Dr. José Gaspar de Francia los maestros privados tuvieron sus primeras intervenciones sociales y se puso en funcionamiento la Escuela Central de Primeras letras. A partir de 1840 y hasta 1870, conocida como la época de los López, el padre Carlos Antonio López y su hijo Francisco Solano López, Paraguay tuvo un importantísimo auge cultural en el que

Aparecieron diarios, revistas, se compuso el Himno Nacional letra y música, la nación tuvo símbolos nacionales, las artes fueron impulsadas. Grupos de estudiantes fueron becados a Europa para conocer los adelantos de la ciencia y tecnología. Se fomentó la educación primaria en las villas y partidos, las escuelas fueron sustentadas por el Estado y los padres de familias, se habilitaron colegios, un Seminario Conciliar, se contrataron profesores extranjeros y se creó una Escuela Normal. (OEI, 1994, p. 3).

Ya en 1870, en la nueva Constitución Nacional de 15 de agosto, bajo las breves presidencias en ese mismo año de Facundo Machaín en primer lugar y la posterior de Cirilo Antonio Rivarola, aparece en su Artículo 8 que "la educación primaria será obligatoria y de atención preferente del Gobierno y el Congreso oirá anualmente los informes que a ese respecto presente el Ministro del ramo para promover por todos los medios posibles la instrucción de los Ciudadanos". La Reforma educativa de 1904 activaba el Plan Franco "en el cual se pormenoriza aspectos referentes al Plan de Estudios del Bachillerato de seis años de duración y aspiraba a proporcionar al estudiante lo que un hombre culto debe conocer, buscando la calidad antes que la cantidad de los conocimientos". (OEI, 1994, p. 4).

La Ley de Educación Obligatoria del 28 de julio de 1909 determinaba que los niños de 7 a 14 años debían recibir la educación primaria en escuelas públicas, privadas o en su domicilio. Se establecieron unos mecanismos de control del absentismo escolar con tal de evitar la desescolarización en ese tramo de edad. Los Jefes políticos estaban obligados a elaborar un censo de niños en edad escolar de su jurisdicción con el fin de informar a las autoridades escolares que emprenderían las acciones necesarias para evitar posibles absentismos. Con la Constitución de 10 de julio de 1940 bajo la presidencia del Mariscal Estigarribia nuevamente encontramos artículos que se refieren a la educación y que plantean algunas novedades respecto a los anteriores:

- Artículo 10.- La educación primaria es obligatoria y gratuita. El Gobierno fomentará la enseñanza secundaria, profesional y universitaria.

- Artículo 11.- El cuidado de la salud de la población y la asistencia social, así como la educación moral, espiritual y física de la juventud, son deberes fundamentales del Estado.

Como observamos, se establece la necesidad de impulsar ya no solamente la educación primaria sino las etapas educativas siguientes como es la secundaria, la técnica o profesional y la universitaria, aunque no confirma su obligatoriedad de ofertarla. Se establecen como deberes del Estado la educación moral, espiritual y física de la juventud que se forma en las escuelas, velando por su cuidado y asistencia. El Estado en esta Constitución asume cada vez más la obligatoriedad de garantizar una educación a los ciudadanos paraguayos que cumpla con los requisitos necesarios para escolarizar y reducir el analfabetismo tanto en las zonas rurales como las urbanas. El profesor 
Ramón Indalecio Cardozo fue el responsable de la siguiente reforma educativa producida en el año 1924

Con el criterio de una enseñanza funcional, inspirada en las actividades básicas del quehacer socioeconómico y en los ideales de libertad y progreso, de rectitud y bien común. Se implantaron en las escuelas normales y elementales, planes y programas en consonancia con los intereses generales de la población. El ciclo se redujo a 6 años de cinco grados, primero inferior, primero superior, segundo, tercero, cuarto y quinto, con énfasis en las asignaturas y actividades fundamentales, trabajos artesanales, práctica agrícola y ganadera, economía doméstica extendida al mantenimiento del huerto familiar y los animales caseros. (OEI, 1994, p. 4).

En 1931 se produjo una nueva reforma educativa bajo la Presidencia de Emiliano González Navero, asumida como Vicepresidente por estar en juicio político el Presidente José Patricio Guggiari, en la que se instauró un novedoso Plan de Estudios para Bachillerato impulsado por el entonces Ministro de Justicia, Culto e Instrucción Pública Dr. Justo P. Benítez que

Mantenía las líneas generales del Plan Franco, en cuanto a lo que respecta a las asignaturas, la diferencia radicó en que prevé un ciclo general de 5 años y un curso de Preparatorio para la Universidad de un año y tiene menos horas de clase. Buscaba una cultura fundamentalmente práctica. Seguramente que la iniciativa más importante y trascendente de la época fue la decisión de organizar la formación magisterial, dando lugar a la creación de las Escuelas Normales. (OEI, 1994, p. 4).

La novedad de esta reforma es que se amplía con más concreción los niveles educativos posteriores como el Bachillerato y la Universidad junto con la reflexión necesaria de formar a los maestros en espacios preparatorios, como las Escuelas Normales, que posibiliten la calidad educativa necesaria para formar a los alumnos. En esta misma línea, ya en 1933 bajo la Presidencia de Eusebio Ayala

Fue aprobado un Plan de Estudios para las Escuelas Normales elaborado por el Profesor Ramón Indalecio Cardozo, que establecía un Curso Preparatorio de un año, más cuatro para obtener el título de Maestro Normal, y tres años más para Profesor Normal. (OEI, 1994, p. 4).

\subsection{LA EDUCACIÓN PARAGUAYA ENTRE 1954 Y LA ACTUALIDAD}

En 1954 se produjo el golpe militar por parte del General Stroessner que mantuvo una dictadura durante más de tres décadas (1954 a 1989) en Paraguay. Esta situación influyó destacablemente en la conformación del sistema educativo paraguayo que tuvo dos fines fundamentales como Rivarola (2000) afirma concluyendo que

La educación fue motivo permanente de preocupación y control de parte de la cúpula de poder por dos razones fundamentales: por una parte, por el reconocimiento de su importancia como un instrumento altamente eficaz de control ideológico y adoctrinamiento. (...) En segundo lugar, el ámbito educativo representaba a la vez un "poder social" importante de controlar y sumar como apoyo del gobierno tanto a través de los múltiples mecanismos de coacción y represión como de los estímulos prebendarios. (p. 10).

No solamente era considerada la educación como un instrumento de control y formación ideológica de la población sino que, además, los docentes eran obligados a formar parte del aparato ideológico del partido del régimen totalitaria.

La exigencia de la afiliación partidaria para tener acceso al cargo de docente, reforzada con una recomendación de un dirigente destacado del partido, del gobierno o de algún miembro prominente 
de las Fuerzas Armadas. Otro recurso sistemáticamente utilizado fue el seguimiento y control de las actividades políticas y sociales de los docentes que se ejercía por medio de los supervisores escolares, en su mayoría militantes activas del partido de gobierno. (...) Contar con la posibilidad o el poder directo de decidir sobre la distribución de los cargos docentes, el manejo de programas o de inversiones varias, adquiría singular relevancia en términos electorales. (Rivarola, 2000, p. 13).

Las faltas o posibles faltas eran muy amplias y ambiguas en su definición, por lo que permitían aplicar a diversas situaciones, creando un clima de temor y subordinación que podía definir si un hecho era o no una falta y si el responsable debía o no ser sancionado. En el artículo 68 se expresa que las infracciones serán penadas con Apercibimiento/Suspensión/Destitución. (Soler, Elías y Portillo, 2015, p. 22).

Esta situación limitaba en gran medida avances en la estructuración del currículo escolar, de la formación pedagógica docente, de la inversión en educación que no superó el 1\% del PIB en numerosas ocasiones y, por ende, de la calidad educativa necesaria para que el país pudiera mejorar socialmente en todos sus ámbitos. La Reforma educativa de 1957 estableció un sistema educativo

Organizado en dos años de preescolar (no obligatorio), seis años de escuela primaria (obligatorio), tres años de ciclo básico (común, especial y vocacional), tres años de bachillerato (bachillerato, comercial, normal y cursos profesionales), dos años de normal superior (continuidad de la formación normal para docentes) y la educación universitaria. (Soler, Elías y Portillo, 2015, p. 18).

Para esta Reforma, el gobierno de Stroessner contó con la colaboración de la UNESCO y del Servicio Cooperativo Americano de Educación (SCIDE) que incorporaron

La elaboración de programas y planes de actividades de estudios para todos los niveles de la educación formal. En lo que respecta a la formación docente, se aprobó un plan de estudios para las escuelas normales en todo el país y se crearon las escuelas normales rurales y las escuelas normales urbanas. A través de un convenio entre los gobiernos de Paraguay y Estados Unidos, se construyó la sede la Escuela Rural Experimental en la ciudad de San Lorenzo. Esta escuela, a cargo del SCIDE, se convirtió en una escuela piloto de formación profesional para obtener el título de Maestro Normal Rural.

La reforma de 1973 fue el resultado de un proceso que se inició con la promulgación de la Constitución Nacional de 1967, un diagnóstico educativo realizado en 1968, la realización del Primer Seminario Nacional sobre Desarrollo Educativo en 1970, la conformación de una comisión encargada de delinear el proyecto de innovaciones educacionales (1971) y la creación del Equipo Técnico de Currículo y Administración Educativa. En 1973, empezó la aplicación gradual del nuevo plan y sus correspondientes programas en el nivel primario en algunas instituciones del país. La reforma de 1973 estuvo vigente hasta el final del gobierno de Alfredo Stroessner y entró en revisión a partir de la transición democrática y de la reforma educativa. (Elías y Segovia, 2011, p. 5)

Fue en el año 1989 cuando tras un periodo de declive económico se produjo un Golpe de Estado por parte del General Andrés Rodríguez quien logró derrocar la dictadura del General Stroessner. De esta forma, "el Golpe del 1989 se termina convirtiendo en otro momento de trascendencia histórica, porque cierra los 35 años de dictadura y abre un período de transición hacia un régimen postautoritario" (Uharte, 2012, p. 23). Rodríguez después del golpe convocó las elecciones a Presidencia del gobierno bajo los preceptos de reunificar el Partido Colorado (el mismo del General Stroessner), la restauración de las Fuerzas Armadas, la garantía y defensa del proceso democrático, defensa del catolicismo y de los Derechos Humanos (López, 2010). Esto supuso un nuevo cambio y desafío no solamente social sino también educativo. En este sentido, no se llegó a aprobar una nueva ley de educación hasta 1998, la Ley General de Educación n 1264 bajo la presidencia del 
mismo general Rodríguez. En la Constitución de 1992, en su Capítulo VII De la educación la Cultura, y en concreto en Artículo 73 "Del derecho a la educación y de sus fines" se establece

Toda persona tiene derecho a la educación integral y permanente, que como sistema y proceso se realiza en el contexto de la cultura de la comunidad. Sus fines son el desarrollo pleno de la personalidad humana y la promoción de la libertad y la paz, la justicia social, la solidaridad, la cooperación y la integración de los pueblos; el respeto a los derechos humanos y los principios democráticos; la afirmación del compromiso con la Patria, de la identidad cultural y la formación intelectual, moral y cívica, así como la eliminación de los contenidos educativos de carácter discriminatorio. La erradicación del analfabetismo y la capacitación para el trabajo son objetivos permanentes del sistema educativo.

De la misma forma distribuye las responsabilidades educativas y las obligaciones del Estado en materia de educación como el respeto a la lengua materna en los primeros años de la escolarización. Como vemos el español y la lengua autóctona como es el guaraní se establecen como lenguas oficiales en la República del Paraguay lo que ayudará sin duda a escolarizar a las minorías étnicas del país, como son las comunidades indígenas guaraníes y, por tanto, a reducir considerablemente los índices de analfabetismo en las zonas no urbanas.

Artículo 75 - La educación es responsabilidad de la sociedad y recae en particular en la familia, en el Municipio y en el Estado. El Estado promoverá programas de complemento nutricional y suministro de útiles escolares para los alumnos de escasos recursos.

Artículo 76 - La educación escolar básica es obligatoria. En las escuelas públicas tendrá carácter gratuito. El Estado fomentará la enseñanza media, técnica, agropecuaria, industrial y la superior o universitaria, así como la investigación científica y tecnológica. La organización del sistema educativo es responsabilidad esencial del Estado, con la participación de las distintas comunidades educativas. Este sistema abarcará a los sectores públicos y privados, así como al ámbito escolar y extraescolar.

Artículo 77 - La enseñanza en los comienzos del proceso escolar se realizará en la lengua oficial materna del educando. Se instruirá asimismo en el conocimiento y en el empleo de ambos idiomas oficiales de la República. En el caso de las minorías étnicas cuya lengua materna no sea el guaraní, se podrá elegir uno de los dos idiomas oficiales.

Con esta base legislativa arrancaba un periodo que, a pesar de las dificultades que iban surgiendo, la educación experimentó unos avances altamente positivos en comparación con periodos anteriores. Rivarola (2006) afirma que "el mejoramiento de la educación paraguaya en el lapso de la transición constituye un fenómeno reconocido y valorado por la sociedad paraguaya, al punto de ser considerada, por la claridad de sus objetivos y continuidad, una manifestación - de hecho única - como política de Estado" (p. 8). De esta forma

Al ser desplazado el régimen autoritario, le tocó a la ciudadanía confrontar dos realidades contrapuestas sobre la educación paraguaya: por una parte, la visión de su papel en el proyecto de construcción de una sociedad radicalmente diferente al que había conformado la dictadura y por otra, las evidencias del tremendo atraso institucional constatado en el sistema educativo y, en un plano más global, del país en su totalidad. (Rivarola, 2006, p. 8).

Los resultados que se obtuvieron fueron, en parte, gracias a la acertada decisión de previamente realizar un diagnóstico de las necesidades educativas del país con el objetivo de partir de un supuesto real para conformar un sistema que atendiera a la realidad educativa del país. Para esta 
tarea se contó con el apoyo y colaboración del Instituto de Desarrollo de la Universidad de Harvard (HIID). Como afirma Rivarola (2006)

El mencionado diagnóstico sirvió de base para la preparación del primer documento doctrinario de la reforma titulado "Desafío Educativo. Una propuesta para el diálogo sobre las oportunidades educativas en el Paraguay", material que a su vez fue el referente directo para la formulación del "Plan Estratégico de la Reforma Educativa". (p. 9).

Entre los objetivos de la Reforma educativa se pretendía dotar de una mejora sustancial de la calidad de los servicios educativos del país en la totalidad de los niveles educativos y modalidades como también asentar los espacios democráticos y reducir ostensiblemente la pobreza. La educación cobraba aquí una especial relevancia en la que los ciudadanos, tras un largo periodo autoritario, habían depositado gran parte de su esperanza. Estas expectativas y funciones otorgadas tras el proceso de Reforma educativa dieron lugar a Ley General de Educación no 1264 del 26 de mayo de 1998. Según la UNESCO (2006) "se institucionalizaron los cambios que el proceso de la reforma educativa incorporo en el sistema educativo nacional, particularmente en relación con la educación general y escolar básica y la administración del Ministerio, que cambia su denominación por el de Ministerio de Educación y Cultura” (p. 7).

En síntesis el sistema educativo paraguayo quedaba estructurado atendiendo a una

Educación de régimen general, la de régimen especial, y otras modalidades de atención educativa. La educación formal se estructura en tres niveles: el primero comprende la educación inicial y la escolar básica, el segundo la educación media, y el tercero la educación superior. La educación inicial comprende dos ciclos (maternal y jardín), mientras que el ciclo preescolar integra la educación escolar básica en forma sistemática, y será obligatorio (...). La educación escolar básica comprende tres ciclos y nueve grados. Es obligatoria y es gratuita en las escuelas públicas de gestión oficial, con la inclusión del preescolar. La educación media comprende el bachillerato o la formación profesional y tiene tres cursos académicos. La formación profesional media está dirigida a la formación en áreas relacionadas con la producción de bienes y servicios. La educación superior (de grado y postgrado) será ordenada por la Ley de Educación Superior y se desarrolla a través de las universidades, institutos superiores (formación docente), y otras instituciones de formación profesional del tercer nivel. (MEC, 1999, citado en UNESCO, 2006, p. 7).

Como observamos se trata de una estructura de un sistema educativo que ya no solo atiende únicamente a los primeros años de escolarización básica sino que otorga importancia a iniciar a edad temprana la educación maternal (hasta los 2 años) No solamente se queda aquí la pionera y novedosa estructura educativa nacional de la historia de Paraguay sino que también ampara y contempla la necesidad de atender a aquellos alumnos con necesidades educativas específicas. La ley respecto a la financiación determina en su Título X, Capítulo 1, Art. 145 que la asignación presupuestaria destinada a la educación nunca podrá ser menor al 20\% de los Presupuestos Generales del Estado de la Nación.

En su Título III distingue entre la educación formal que correspondería a la educación inicial y básica en su primer nivel, la media y la formación profesional media en su segundo y la educación superior como tercer nivel. Incorpora dentro del mismo título pero en la sección III la educación de postgrado, la educación no formal, la educación refleja (colaboración de los medios de información y comunicación social), la educación a distancia y distingue entre educación privada y educación pública. En su Título IV incluye la educación de régimen especial integrada por la educación artística, arte dramático, música y danza, educación en lenguas extranjeras y otras etnias. 
Ya en su Título V determina las modalidades de atención educativa según la singularidad de los beneficiarios estableciéndose la educación general básica y la educación permanente, la educación para grupos étnicos, la educación campesina y rural, la educación para personas con limitaciones o con capacidades excepcionales, la educación para la rehabilitación social y prevención de adicciones. Termina este mismo título con la modalidad de educación militar y educación policial junto con la educación para ministros de culto de las iglesias y comunidades religiosas reconocidas. (MEC, 1998) No es objeto de este artículo profundizar en un análisis de cada uno de los apartados de la Ley General de Educación n 1264, hoy vigente con algunas modificaciones y reformas, pero sí al menos mostrar las líneas maestras de atención de un sistema educativo que nació con la idea de impulsar una democracia que atienda a sus ciudadanos en sus necesidades de alfabetización y en igualdad de oportunidades. Se trata de una ley muy ambiciosa que requiere de un apoyo financiero adecuado a las necesidades educativas y a una renovación amplía de los procesos de implementación hasta ahora seguidos. A ello cabe sumar la esencial atención a la calidad formativa de los docentes de los diferentes niveles educativos y a su capacidad de adaptación ante las nuevas realidades sociales de Paraguay. Desde este punto de vista Rivarola (2000) afirma que

La reforma educativa actualmente en ejecución ha distinguido una serie de líneas de acción en lo relativo al mejoramiento de la calidad de la educación paraguaya. En dicha cuestión, una de las preocupaciones principales ha sido el bajo nivel de logros constatado, primordialmente en la esfera de la educación escolar básica. (...) buscando minimizar la incidencia de los factores que incurren negativamente sobre el fenómeno del rendimiento educativo. (p. 20)

Los factores que inciden negativamente en la calidad de la educación y en la consecución de las metas educativas pueden definirse entorno a los textos escolares, el tiempo efectivo de las actividades en el aula, la condición lingüística respecto al guaraní y al castellano, la reforma curricular y la formación docente. (Rivarola, 2000). Estas observaciones nos permitirán analizar en el siguiente apartado la confluencia entre lo que debe mejorarse y lo que se ha mejorado en el sistema educativo paraguayo en estos últimos 26 años de vigencia de la actual ley educativa y el acuerdo de desarrollo del programa Metas Educativas 2021. La Ley General de Educación ha sido complementada con nuevos marcos normativos destacando los más actuales como La Ley n ${ }^{\circ} 6102$ que modifica el numeral 1 del artículo 12 de la ley no 4758/12 "que crea el fondo nacional de inversión pública y desarrollo (FONACIDE) y el fondo para la excelencia de la educación y la investigación" de julio de 2018, Ley n 6103 de junio de 2018 que crea el Programa Nacional de Atención Integral a los Trastornos del Espectro Autista (PNAITEA) para el abordaje integral e interdisciplinario y la protección social de las personas que presentan Trastornos del Espectro Autista (TEA), la Ley no 5749 de enero del 2017 que establece la Carta Orgánica del Ministerio de Educación y Ciencias, la Ley n ${ }^{\circ} 5778$ de diciembre del 2016 que crea el programa nacional de apoyo a la estimulación oportuna (PRONAES) o la Ley n 5347 de noviembre del 2014 que dispone el libre acceso de postulantes indígenas a las carreras de nivel terciario habilitadas tanto en universidades públicas como en universidades privadas.

\section{PARAGUAY Y LAS METAS EDUCATIVAS 2021}

La revisión realizada del proceso que ha vivido el sistema educativo paraguayo desde su independencia de España en 1811 nos ha permitido determinar los precedentes que han llevado a Paraguay a comprometerse desde el 2010 en la búsqueda de soluciones a los retos educativos a los que se enfrenta y desde donde partía. Este compromiso se oficializó en la XX Conferencia Iberoamericana de Ministros de Educación y en la XX Cumbre Iberoamericana de Jefas y Jefes de Estado y de Gobierno los países que se adhirieron al proyecto "Metas Educativas 2021”, año muy 
señalado para Iberoamérica ya que coincide con el fin de las celebraciones de los bicentenarios de la independencia. En la declaración final de esta Cumbre

Se subrayaba que el desafío asumido en esta celebración del Bicentenario radica en redoblar y hacer más eficientes los esfuerzos en aras de alcanzar el ineludible objetivo de atender y concluir las tareas pendientes para lograr una educación con inclusión social intra e intercultural en la región iberoamericana, de calidad para todos y todas, para promover una Iberoamérica más justa, con desarrollo económico, social y cultural en el marco de sociedades democráticas, solidarias y participativas que promuevan el bienestar de todos los habitantes de nuestra región. (Marchesi, 2011, p. 13).

En este marco referencial Paraguay se sumó a las directrices de este programa que tuvo un primer informe de base en el mismo 2011 recogiendo datos de varios años anteriores. Esta decisión posibilitó conocer a cada país cuál era su realidad educativa particular en aras a iniciar el proceso de mejora en el marco del programa acordado. Veamos a continuación la comparativa en Paraguay de las metas educativas de origen en 2011, las alcanzadas en 2015 y la situación actual en 2018 a tres años vista del plazo de compromiso para su cumplimiento. Iremos a desgranando cada una de las once metas generales acordadas. Analicemos a continuación los datos contextuales de cada año.

Atendiendo a los datos del Banco Mundial recogidos en 2011, Paraguay tenía una población de 6.460.000 habitantes, correspondiendo un 59\% a población urbana y situándose el porcentaje de población entre 15 y 64 años en un 62\%. Según informe del Programa de las Naciones Unidas para el Desarrollo (PNUD) el Índice de Desarrollo Humano (IDH) en Paraguay era del 0,665 ocupando el lugar 107 de 187 países situándose en la categoría de desarrollo humano medio. La esperanza de vida al nacer estaba en los 72,5 años, los años de promedio de escolaridad estaba en los 7,7 siendo 12,1 años los esperados de escolarización. El Ingreso Nacional Bruto (INB) per cápita estaba en 4.727 dólares. Respecto a la población bajo la línea de pobreza de 1,25 dólares por día se situaba en el periodo del 2000 al 2009 en el 5,1\%. La población en riesgo de sufrir pobreza o extrema pobreza en 2011 era del 15,1\% y un 6,1\% respectivamente, indicadores que describen la alarmante situación social en 2011 respecto al promedio mundial ante la cual la educación suponía una esperanza de mejora. En el ámbito educativo el informe recoge la medición del periodo del 2005 al 2010 respecto a la tasa de alfabetización adulta (15 años y mayor) en un 94,6\%. En Educación Primaria era del $99,6 \%$, de un $66,8 \%$ en Secundaria y en la Terciaria de un 36,5\%. Como observamos valores preocupantes respecto a la Secundaria y Terciaria que se convertirán, como veremos más adelante, en una de las metas educativas más importantes a conseguir por Paraguay para el 2021. El gasto público en educación respecto al PIB fue del 7,1\% en el periodo del 2006 al 2009. (ONU, 2011).

En el año 2015 Paraguay aumentó su población hasta los 6.639.120 habitantes, correspondiendo a un $60 \%$ a población urbana, produciéndose un incremento de un $1 \%$ respecto a 2011 y manteniéndose en 2017 (últimos datos del Banco Mundial disponibles) con ese mismo 60\%. Respecto a la población entre los 15 y 64 años se sitúan en 2015 y en 2017 en un 64\%, datos que indican un aumento de la población en ese tramo de edad y que Paraguay precisa tener en cuenta para implementar mejoras en los porcentajes de asistencia y finalización en la Educación Secundaria y la Terciaria como marca la Meta Educativa Cuarta. El informe del PNUD de 2016 que recoge los datos del año 2015, rebaja la posición de Paraguay respecto a su IDH del 107 al 110 lo que indica que aunque mejoró en ese periodo del 2011 al 2015 estableciéndose en un 0,693 perdió posiciones respecto a otros países que mejoraron más. En el informe se distingue entre hombres y mujeres respecto a los porcentajes de los distintos indicadores. De esta forma La esperanza de vida al nacer se situaba en los 73 años. Los años de promedio de escolaridad estaba en los 8,1 años 
produciéndose un ligero aumento respecto al anterior informe. Los años esperados de escolarización era de 12,3 con un mínimo aumento respecto al 2011. El Ingreso Nacional Bruto (PIB) per cápita estaba en 8.182 dólares doblando casi la cifra del 2011. Respecto a los índices de pobreza en este informe no se detallan por falta de información de origen. En el ámbito educativo el informe recoge la medición del periodo del 2005 al 2015 respecto a la tasa de alfabetización adulta (15 años y mayor) en un 95,6\% aumentando en un punto respecto a 2011. En Educación Primaria era del $106 \%$, de un 77\% en Secundaria y en la Terciaria de un 35\% en el periodo del 2010 al 2015. Los valores siguen siendo preocupantes respecto a la Secundaria y Terciaria, observándose una disminución de un punto y medio respecto a la Terciaria. El gasto público en educación se quedaba en un 5\% del PIB en el periodo 2010-2014 rebajándose en un 2,1\% respecto al informe del 2011, dato necesariamente a tener en cuenta por su bajo porcentaje. Estos son los últimos datos que se recogen del informe de 2016 puesto que no ha habido una nueva actualización con otro informe hasta la fecha. Conocido el contexto donde se adscriben las once metas educativas acordadas para 2021, teniendo en cuenta la extensión de las mismas hemos considerado oportuno profundizar en el análisis de tres de ellas cuyos resultados los veremos a continuación. (ONU, 2016).

\subsection{META GENERAL 1. REFORZAR Y AMPLIAR LA PARTICIPACIÓN DE LA SOCIEDAD EN LA ACCIÓN EDUCADORA.}

Hemos escogido estas tres metas por su carácter social, institucional y docente atendiendo a los ámbitos más cercanos a la educación, conociendo su evolución y su forma de constatar esos avances a la par que el análisis de las 11 metas superaría los límites de extensión de este artículo. Esta meta pretende que los países incentiven y aumenten la participación de los diferentes agentes sociales de forma coordinada a través de proyectos educativos. En el caso de Paraguay en la Ley General de Educación de 1998, en su Art. 127 establece que las organizaciones estudiantiles de educación escolar básica y media se regirán por estatutos aprobados por las autoridades de la institución, considerado como una restricción que posteriormente con la Ley n 3.488 de 29 de mayo de 2008 este mismo artículo fue derogado quedando de la siguiente forma:

Artículo $1^{\circ}$.- Modifícase el Artículo 127 de la Ley No 1264/98 “General de Educación”, cuyo texto queda redactado de la siguiente manera:

Art. 127.- Los estudiantes de educación escolar básica y media que quieran constituir una organización estudiantil, expresarán su voluntad mediante estatutos, cuyas cláusulas estarán acordes con la Constitución Nacional, el Código Electoral y el Código Civil, en lo referente a las asociaciones de utilidad pública, en cuanto le sea aplicable. La conformación de una organización estudiantil será notificada de inmediato a las autoridades de la respectiva institución educativa, adjuntando un ejemplar del estatuto de la organización. (MEC, 2008, p. 1).

Observamos que es una buena iniciativa para permitir la participación de los estudiantes en los centros educativos y en otros ámbitos sociales con el fin de que sus propuestas puedan ayudar a avanzar en aspectos como la educación desde un punto de vista democrático. Aunque fue en el 2008 se trata de un ejemplo del cambio que estaba experimentando la sociedad paraguaya después de una larga dictadura. Actualmente los espacios de participación de los estudiantes se vertebra a través de los Consejos Departamentales de Educación, los Consejos Distritales de Educación, Equipo de Gestión de Instituciones Educativas, las Asociaciones de Cooperación Escolar que analizaremos a continuación y en las organizaciones estudiantiles como los Centros de Estudiantes, los Consejos de Delegados Institucional y los Consejos Escolares, los Clubes, Academias y Cooperativas de Estudiantes. 
No encontramos hasta el 2013 una iniciativa que refuerce la perspectiva de logro de esta meta con la Ley $\mathrm{n}^{\circ} 4853$ por la que se regula la conformación, organización y funcionamiento de la Asociación de Cooperación Escolar (ACE) en las instituciones educativas del país. En el Capítulo 1 se le define como organismo de cogestión, apoyo y colaboración en las instituciones educativas de Gestión Oficial, Privada y Privada Subvencionada de los niveles de Educación Inicial, Escolar Básica y Educación Media o Secundaria. Su equiparación española podría establecerse como la Asociación de Madres y Padres de Alumnos (AMPA). Respecto a su concepto, principios, conformación y domicilio la ley establece

Artículo $3^{\circ}$.- La Asociación de Cooperación Escolar (ACE) promoverá como principios rectores de su vida institucional: la participación democrática, la justicia social, la inclusividad, la solidaridad, equidad de género, respeto y transparencia en la gestión y la no discriminación en el ámbito educativo escolar, desde la perspectiva de los Derechos Humanos.

Artículo $4^{\circ}$.- (...) será integrada únicamente por padres, madres, encargados o tutores de alumnos matriculados en la institución educativa correspondiente.

Artículo $5^{\circ}$.- (...) se constituye, con el objeto de cooperar con el Estado en forma participativa y organizada a fin de contribuir al desarrollo y mejoramiento de la calidad de la educación.

Artículo $6^{\circ}$.- Para todos los efectos legales, (...) fijará domicilio en la institución educativa donde coopera. (MEC, 2013, pp. 1-2)

En su Capítulo V determina que la ACE tendrá como autoridades que gestionen su funcionamiento la Asamblea General y la Comisión Directiva bajo esta estructura con mención a las comunidades indígenas en su Capítulo VIII:

Artículo 16.- La Asamblea General es la máxima autoridad (...). Ella debe ser convocada en la forma y tiempos determinados por sus estatutos sociales o cuando la solución de asuntos urgentes de su competencia lo exija, o a petición escrita de por los menos la quinta parte de los asociados.

Artículo 17.- La Asamblea General Ordinaria será realizada entre los 90 (noventa) y 120 (ciento veinte) días posteriores al cierre del año civil anterior, para cuya convocatoria se someterá a las disposiciones establecidas en los estatutos sociales y al Código Civil.

Artículo 18.- La Comisión Directiva (...) estará compuesta por un número mínimo de 5 (cinco) miembros y un número máximo de 15 (quince) miembros, designados conforme a las reglas establecidas en sus estatutos sociales. Deberá estar integrada por lo menos por un presidente, un vicepresidente, un tesorero, un secretario y un vocal.

Artículo 26.- En las comunidades indígenas, (...) se subordinará a las formas de organización y participación de los pueblos indígenas garantizados por la Constitución Nacional y establecidos en las disposiciones legales y administrativas que regulan la materia. (MEC, 2013, pp. 7-9).

En esta misma línea, ya en la Ley General de Educación no 1264 se determinaba en su Título IX "Los establecimientos educativos" y Capítulo II Art. 143 que la dirección del centro escolar fomentará la asociación de padres y la de alumnos como también la de educadores y de personal administrativo y auxiliar de ese mismo centro dentro del marco democrático. (MEC, 1998). Se establece una clara alusión para que la sociedad participe también la acción educadora implicándose en los procesos y toma de decisiones junto a las autoridades gubernamentales. 
Otra de las iniciativas que podemos destacar en la web del Ministerio de Educación y Ciencias de Paraguay es el Programa Social en Educación "Por nuestras escuelas" del 2014 cuyo objetivo es promover la articulación entre los actores locales y la comunidad educativa en torno a los requerimientos de la escuela que permitan garantizar las mejores condiciones de aprendizaje para las y los estudiantes. Entre los proyectos se destaca el proyecto enredados cuyo objetivo es promover espacios para el voluntariado en función a la relación de la escuela con el entorno local; el proyecto escuelas saludables en el que el objetivo es promover la mejora de los ambientes de aprendizaje que garantice el derecho a una educación en condiciones de dignidad como por ejemplo el acceso a agua potable; el proyecto conectados cuyo objetivo es Incorporar materiales educativos y equipamiento tecnológico con conectividad a instituciones educativas de tercer ciclo y educación media en contextos de vulnerabilidad; y el proyecto educarse con el objetivo de ampliar las oportunidades de educación a lo largo de la vida para empleados y/o funcionarios de empresas y organizaciones.

\subsection{META GENERAL 8. FORTALECER LA PROFESIÓN DOCENTE.}

Entre los objetivos que propone esta meta es la mejorar la formación inicial, generalmente universitaria, de los docentes de los niveles educativos de Educación Primaria y de Educación Secundaria con una acreditación oficial de su calidad que actualmente está siendo realizada por parte de la Agencia Nacional de Evaluación y Acreditación de la Educación Superior (ANEAES). Exige a su vez que los evaluadores que determinen esa calidad a través de la acreditación tengan la formación pedagógica y las competencias necesarias para garantizar el proceso. Otros de los objetivos prioritarios de esta meta es favorecer la capacitación continua y el desarrollo de la carrera profesional docente.

Rivarola (2000) ya apuntaba que era necesario

Aumentar las exigencias para la selección de aspirantes a la profesión docente, el fortalecimiento de la formación inicial en las prácticas pedagógicas y la incorporación de profesionales de otras áreas como docentes del bachillerato. (...) Los márgenes de mejoramiento observados en la preparación de los maestros no satisfacen las expectativas sobre las metas previstas. Esto ha dado razón a muchos analistas que han enfatizado que, a pesar del esfuerzo aplicado en el perfeccionamiento de los mismos, tanto en el orden curricular como en la práctica de aula, tales reformas no han producido el cambio esperado. Tal situación supone (...) reorientar e intensificar los programas de capacitación y establecer políticas de estímulos para alcanzar un mayor grado de involucramiento de los maestros en la tarea de enseñanza. (p. 23).

Actualmente aunque hay establecido un proceso de selección reglamentado para las oposiciones y concurso de méritos sigue siendo insuficiente puesto que la exigencia no ha aumentado, la capacitación sigue estando desajustada a las necesidades reales y conocimientos previos de los docentes en algunos casos, las políticas de estimulación siguen siendo escasas y, entre otras, la estabilidad docente tanto económica como académica es deficitaria pero mejorable con las políticas que se están desarrollando. En el informe del Plan Nacional 2024 publicado por el MEC (2011) se evidencia que

La política vigente sobre las estructuras e instituciones para la formación docente inicial y en servicio esta manifestando claramente síntomas de agotamiento. El vertiginoso crecimiento de nuevos institutos de formación docente desde el inicio de la reforma educativa hasta hace dos o tres años ha entrado en crisis con el cierre de muchos de ellos y la constatación de la baja calidad de su servicio de formación, evidenciado en el insuficiente nivel de competencias de la mayoría de sus egresados/as. 
(...). Ha entrado en crisis la demanda para la carrera ante la saturación del mercado. Se desea y se necesita mejorar la calidad de la mayoría de los institutos de formación docente. Urge un cambio profundo de los currículos de la formación inicial y una revisión de las experiencias de formación en servicio para los y las profesionales que están en ejercicio. Se plantea la posibilidad de levantar el nivel académico y profesional de la formación. (p. 19).

En el 2011 la política de formación docente se convirtió en uno de los pilares y desafíos a los que debía enfrentarse el Ministerio de Educación y Cultura de aquella época que coincidía con el inicio del Programa Metas Educativas 2021. Aunque se ha avanzado mucho en esta necesidad, las oposiciones a docente ofrecen plazas fijas, pero en su mayoría, con horas semanales en distintos colegios hasta completar jornada laboral. Los docentes tienen que desplazarse a otros colegios el mismo día con las consecuencias que se derivan de esta inestabilidad. Quizás se debería hacer un proceso de selección más transparente en el que además de explicar este mismo proceso de selección, se establezca previamente una baremación por los méritos, se publiquen las asignaciones de plazas con la puntuación obtenida dando la posibilidad de alegaciones a los candidatos y se agrupen las horas semanales en un mismo colegio.

Según datos del Ministerio de Educación y Ciencias, en el 2017 se ofertaron 23 cursos con un total de 38.538 plazas para educadores. Esta misma fuente afirmaba que se había alcanzado la meta prevista del programa formativo fijada en cubrir el $72 \%$ del total de los 53.342 educadores. Para el 2018 son 26.680 educadores los que se han matriculado en 12 nuevos cursos en las modalidades de actualización, técnico docentes y especialización. Es muy importante que los docentes puedan recibir la capacitación necesaria para afrontar los nuevos desafíos educativos y así atender las necesidades de la comunidad escolar pero también es esencial que está formación tenga su reflejo práctico en el aula. Es necesario establecer los mecanismos e instrumentos de evaluación adecuados, como realizar pruebas de nivel a los alumnos cada dos o tres años, que permitan garantizar que esa formación docente tiene su efecto esperado y no se convierte en una mera aglutinación de títulos formativos sin que se lleve a la práctica lo aprendido. Al igual que en España sería interesante que en Paraguay se iniciaran procesos de seguimiento y evaluación docente regulares en el aula con el fin de lograr que quienes sean docentes lo hagan por vocación y no por otros intereses personales. Los mejores docentes son los que deben formar a los ciudadanos si se espera que un país prospere.

Una excelente iniciativa es el documento del MEC de 2016 "Propuesta de reglamentación de la carrera profesional del educador" cuyos objetivos son: "regular el ingreso, promoción, evaluación del desempeño, seguimiento, permanencia y retiro del educador y la educadora profesional, en el sistema educativo paraguayo, como también fortalecer el desarrollo profesional del educador, para que impacte en mejores niveles de desempeño pedagógico y en el logro de aprendizajes significativos de los estudiantes". (MEC, 2016, p. 5). Sin duda se trata de una aportación más de cara a la mejora de las políticas educativas de formación y regulación de la profesión docente que tanto precisa Paraguay.

\subsection{META GENERAL 11. EVALUAR EL FUNCIONAMIENTO DE LOS SISTEMAS EDUCATIVOS Y DEL PROYECTO “METAS EDUCATIVAS 2021”.}

Se pretende que los países firmantes creen los organismos necesarios para evaluar no solamente la marcha del sistema educativo propio sino también los sistemas de planificación, las unidades de estadística, el avance o logro sobre las metas pactadas dentro del programa y la participación de los diferentes sectores sociales en la supervisión de este proyecto con la elaboración de un informe cada dos años. En la web del MEC y otras fuentes consultadas encontramos solamente un boletín 
informativo sobre las mejoras educativas del 2013 y de estadísticas educativas del 2011. No así alguno no que hiciera referencia a los avances sobre el programa Metas Educativas 2021.

Respecto a la creación de instituciones de evaluación Paraguay ha cumplido con sus deberes al crear, entre otros, el Sistema Nacional de Evaluación del Proceso Educativo (SNEPE), el Instituto Nacional de Evaluación Educativa (INEE), el Consejo Nacional de Educación y Ciencias (CONEC) y la Agencia Nacional de Evaluación y Acreditación de la Educación Superior (ANEAES) que podrán contribuir para la elaboración de los informes del Instituto de Seguimiento y Evaluación de las Metas Educativas (IESME) y de su Consejo Rector de la OEI. El último informe elaborado por este Instituto es "Miradas sobre la Educación en Iberoamérica" de 2016 con la participación de Paraguay a través de la Dirección General de Planificación Educativa, de la Directora de Estadística Educativa y de la Jefa del Departamento de Estadística todos ellos pertenecientes al Ministerio de Educación y Cultura del momento.

El SNEPE se crea en Paraguay en 1995, estando vigente actualmente, con el propósito de generar información permanente, válida, confiable y oportuna sobre el nivel de logro académico de los niños y jóvenes paraguayos, así como de los factores asociados o contextuales que puedan ayudar a interpretar los resultados a modo de diagnóstico. Desde su instalación, el SNEPE tuvo como propósito apoyar la toma de decisiones en relación a políticas educativas con fines de mejora.

La Ley n 5749 en la se establece la Carta Orgánica del Ministerio de Educación y Ciencias del 2017 define el INEE en su Capítulo V “Órganos misionales” y su Art. 50 como

Órgano con autonomía funcional y académica que tiene a su cargo la responsabilidad de implementar el Sistema Nacional de Evaluación de la calidad de la Educación en sus diferentes componentes constitutivos. (...) tendrá el nivel jerárquico de Dirección General y dependerá del Ministro de Educación y Ciencias. (MEC, 2017, p. 21).

Por su parte, en esta misma ley se determina en su Capítulo III “Órganos consultivos” y su Art. 22 que el CONEC es el "órgano responsable de proponer las políticas educativas, la permanente mejora del sistema educativo nacional y acompañar su implementación en la diversidad de sus elementos y aspectos concernientes" (MEC, 2017, p. 9).

La Agencia Nacional de Evaluación y Acreditación de la Educación Superior fue creada en el año 2003 bajo el amparo de la Ley no 2072 “con la finalidad de evaluar y en su caso, acreditar la calidad académica de las instituciones de educación superior que se someten a su escrutinio y producir informes técnicos sobre los requerimientos académicos de las carreras y de las instituciones de educación superior" (MEC, 2003, p. 1) De esta forma, la agencia contribuirá a esta conformación de la calidad educativa evaluando y acreditando la calidad de la educación superior que reciben, entre otros, los futuros docentes con el fin de mejorarla.

Otro de los instrumentos interesantes es el Manual Instructivo para la Evaluación del Desempeño de los docentes del primer y segundo ciclos de la Educación Escolar Básica de las Instituciones de gestión oficial. Tiene su Resolución n ${ }^{\circ} 26707$ de 1 de diciembre de 2016. Junto con el reglamento de la carrera profesional del educador constituyen dos pilares básicos que ofrecen ciertas garantías de evaluación de la profesión docente. No obstante, sería necesario revisar el proceso que se sigue en las aulas para su implementación, la formación de los evaluadores (pares académicos y Comisión Evaluadora), el sesgo en la recogida de datos, la validez de lo presentado como resultado, el conflicto de intereses y las acciones que se tomen en pro de premiar la excelencia (como buen 
incentivo de mejora docente) y gestionando la ayuda al docente de cara a su perfeccionamiento en caso de resultados no satisfactorios.

\section{CONCLUSIONES}

Aunque los avances han sido numerosos respecto a las metas educativas que hemos analizado con la promulgación de leyes, convenios y decretos consideramos las limitaciones de este artículo para constatar los resultados de lo promulgado por la falta de informes evaluativos actuales elaborados por el MEC de Paraguay. Aun así consideramos que lo tratado en este artículo nos ha ofrecido una visión amplia de los cambios en materia de educación que está experimentando Paraguay cuyos beneficios se podrán recoger en un corto espacio de tiempo. No obstante, es conveniente señalar que una de las metas que lleva menos desarrollo es la meta 8 referida al fortalecimiento de la profesión docente. Hemos observado que el sistema de selección de los docentes actual, a pesar de que existe un proceso de oposición y méritos de acceso a la profesión, no se describe con claridad las fases del examen de oposición, la baremación de los méritos y la adjudicación de las plazas, además de precisar una necesaria actualización en su reglamento acorde a las nuevas necesidades y perfiles actuales ya que el último aprobado data del 10 de febrero del 2012 que corresponde, a su vez, a una actualización del 2009. Los docentes precisan de un mayor reconocimiento social y económico que ofrezca la base suficiente para garantizar una calidad educativa tan explícita en las leyes analizadas y en la misma Constitución de 1992.

Sería muy interesante que se garantizara un sueldo acorde a la exigencia y responsabilidad personal y profesional junto con la permanencia en un centro educativo concreto sin tener que ir sumando horas de un colegio a otro afectando al tiempo de dedicación tanto en la formación del docente como, probablemente, en la preparación de las clases. Los niveles de calidad ascenderían al ofrecer una estabilidad económica y académica al docente sin tener que suspender clases, como hemos leído en algunos documentos oficiales, para que asista a cursos de formación. Quizás uno de los nuevos elementos en pro de la consecución de la calidad educativa sea la acreditación de las buenas prácticas de los maestros mediante la integración de un proceso evaluativo procesual que incentive la excelencia y la vocación docente. También cabría integrar procesos evaluativos que nos muestren la coordinación positiva entre la familia y la escuela como ejes principales de la calidad educativa. Integrar el reconocimiento al esfuerzo y la excelencia de los maestros sería una posible nueva medida de incentivo además de afianzar su autoridad y dignificación. El actual sistema funcionarial público de la educación paraguayo debe actualizarse para atender de forma eficaz y eficiente a los desafíos del siglo XXI. La mejor forma de garantizar la longevidad de un sistema educativo y su consecuente proceso de calidad, es promocionando un pacto de Estado responsable entre los partidos políticos que tienen representación en el Congreso Nacional como compromiso indisoluble con la ciudadanía. Con todo ello Paraguay está en el buen camino hacia la consecución del logro del programa Metas Educativas 2021 aunque sería necesario poder constatarlo con la realidad a través de informes anuales o bianuales por parte de las agencias evaluativas creadas, haciéndolos accesibles en los espacios oficiales correspondientes. 


\section{BIBLIOGRAFÍA}

Almeida, E., Bastos, F., Quijada, A. y Acevedo, M. C. (2018). Paraguay: Rutas para el desarrollo. Banco Interamericano para el Desarrollo.

Elías, R., y Segovia, E. (2011). La educación en tiempos de Stroessner. En La educación en el Paraguay independiente (págs. 173-206). Asunción: Ministerio de Educación y Cultura (MEC).

López, M. (2010). La democracia en Paraguay. Revista Enfoques, 13, 89-106.

Marchesi, A. (2009). Las Metas Educativas 2021. Un proyecto iberoamericano para transformar la educación en la década de los bicentenarios. Revista Iberoamericana de Ciencia, Tecnología y Sociedad, $12(4), 87-157$.

Marchesi, A. (2011). Miradas sobre la educación en Iberoamérica: Metas educativas 2021. Madrid: Organización de Estados Iberoamericanos para la Educación, la Ciencia y la Cultura (OEI).

Ministerio de Educación y Cultura (MEC). (2011). Plan Nacional de Educación 2024. Hacia el centenario de la Escuela Nueva Ramón Indalecio Cardozo. Asunción: MEC.

Ministerio de Educación y Cultura (MEC). (2012). Reglamento Concurso Público de Oposición para la Selección de Educadores. Asunción: MEC.

Ministerio de Educación y Cultura (MEC). (2014). Programa Social en Educación "Por nuestras escuelas". Asunción: MEC.

Ministerio de Educación y Cultura (MEC). (2016). Propuesta de reglamentación de la carrera profesional del educador. Asunción: MEC.

Ministerio de Educación y Cultura (MEC). Ley General de Educación nº 1264 del 14 de mayo de 1998. Asunción: MEC.

Ministerio de Educación y Cultura (MEC). Ley n 2072 de creación de la Agencia Nacional de Evaluación y Acreditación de la Educación Superior del 13 de febrero de 2003. Asunción: MEC.

Ministerio de Educación y Cultura (MEC). Ley n 3488 que modifica el artículo 127 de la Ley n 1264/98 General de Educación del 29 de mayo de 2008. Asunción: MEC.

Ministerio de Educación y Cultura (MEC). Ley n 4853 por la que se regula la conformación, organización y funcionamiento de la Asociación de Cooperación Escolar (ACE) de 21 de febrero de 2013. Asunción: MEC.

Ministerio de Educación y Cultura (MEC). Ley n $n^{\circ} 547$ dispone el libre acceso de postulantes indígenas a las carreras de nivel terciario habilitadas tanto en universidades públicas como en universidades privadas del 10 de noviembre de 2014. Asunción: MEC.

Ministerio de Educación y Cultura (MEC). Ley n 5749 que establece la Carta Orgánica del Ministerio de Educación y Ciencias del 24 de enero de 2017. Asunción: MEC. 
Ministerio de Educación y Cultura (MEC). Ley n 5778 que crea el programa nacional de apoyo a la estimulación oportuna (PRONAES) del 19 de diciembre de 2016. Asunción: MEC.

Ministerio de Educación y Cultura (MEC). Ley n 6102 que modifica el numeral 1 del artículo 12 de la ley $n^{\circ}$ 4758/12 que crea el fondo nacional de inversión pública y desarrollo (FONACIDE) y el fondo para la excelencia de la educación y la investigación" del 28 de junio de 2018. Asunción: MEC.

Ministerio de Educación y Cultura (MEC). Ley no 6103que crea el programa nacional de atención integral a los trastornos del espectro autista (PNAITEA) para el abordaje integral e interdisciplinario y la protección social de las personas que presentan trastornos del espectro autista (TEA) del 29 de junio del 2018. Asunción: MEC.

Organización de las Naciones Unidas (ONU). (2011). Informe sobre Desarrollo Humano 2011. Sostenibilidad y equidad: Un mejor futuro para todos. EE.UU.: Programa Naciones Unidas para el Desarrollo (PNUD)

Organización de las Naciones Unidas (ONU). (2016). Informe sobre Desarrollo Humano 2016. Desarrollo humano para todos. EE.UU.: Programa Naciones Unidas para el Desarrollo (PNUD)

Organización de los Estados Iberoamericanos para la Educación, la Ciencia y la Cultura. (OEI). (2016). Miradas sobre la educación en Iberoamérica. Avances en las Metas Educativas 2021. Madrid: OEI.

Organización de los Estados Iberoamericanos para la Educación, la Ciencia y la Cultura. (OEI). (1994). Breve evolución histórica del sistema educativo. Paraguay. Recuperado de https://www.oei.es/historico/quipu/paraguay/index.html

Rivarola, D. M. (2000). La Reforma educativa en el Paraguay. Chile: Naciones Unidas. CEPAL.

Rivarola, D. M. (2006). Paraguay, reforma educativa y crisis de la docencia. Asunción: CPES.

Soler, L., Elías, R. y Portillo, A. (2015). El régimen stronista y su incidencia en la configuración y las prácticas escolares (1954-1970). Revista Cuadernos Chilenos de Historia de la Educación, 4, 10-33.

Uharte Pozas, L. M. (2012). El proceso de democratización paraguayo: avances y resistencias. Revista América Latina Hoy, 60, 17-42.

UNESCO (2006). Principios y objetivos de la educación en Paraguay. Recuperado de http://www.ibe.unesco.org/fileadmin/user_upload/archive/Countries/WDE/2006/LATI N_AMERICA_and_the_CARIBBEAN/Paraguay/Paraguay.htm 


\section{SOBRE EL AUTOR}

\section{Carlos Novella García}

Carlos Novella García es Licenciado y Doctor en Pedagogía, Máster Universitario en Ética y Democracia. Fue profesor de la Universitat de València y desde hace 11 años profesor de Magisterio, Pedagogía y Educación Social de la Universidad Católica de Valencia San Vicente Mártir. Asesor técnico pedagógico de la Coordinación Departamental de Inspecciones Educativas del Guairá. (Paraguay) hasta 2017. Coordinador Programa Internacional de Voluntariado en educación en Paraguay desde 2007. Profesor Honorario de la Universidad Nacional de Villarrica del Espíritu Santo (Paraguay). Publicaciones en el área de los métodos pedagógicos, la pedagogía comparada y la pedagogía agustiniana. Líneas de investigación como la ética política, políticas educativas, filosofía política, educación comparada y pedagogía agustiniana.

Contact information: carlos.novella@ucv.es

Universidad Católica de Valencia San Vicente Mártir. 